\title{
Analysis of Wheat Straw Biodiversity for Use as a Feedstock for Biofuel Production
}

\author{
Yifat Tishler $^{1,2} \cdot$ Aviva Samach $^{1} \cdot$ Ilana Rogachev ${ }^{1} \cdot$ Rivka Elbaum $^{2} \cdot$ Avraham A. Levy $^{1}$
}

Published online: 16 June 2015

(C) The Author(s) 2015. This article is published with open access at Springerlink.com

\begin{abstract}
Wheat straw is a potential source of feedstock for biofuel production that does not compete with food. We have screened 48 wheat lines from a collection representing a broad range of the biodiversity of wild and domestic wheat. Wheat straw was fractionated into water-soluble and nonsoluble fractions. In the water-soluble fraction (WSF), we found a broad variation in the concentration of free soluble sugars (FSS) and a narrow variation in starch. The FSS fraction could reach levels of reducing sugars as high as $130 \mathrm{~g}$ sugar $/ \mathrm{kg}$ of straw. The analysis of the FSS by ion chromatography indicated that fructose and glucose were the major sugar monomers in this fraction. The composition of the nonsoluble cell wall fraction was determined by both pyrolysis and direct chemical analysis. These analyses showed a limited variation in the lignin or
\end{abstract}

Electronic supplementary material The online version of this article (doi:10.1007/s12155-015-9631-0) contains supplementary material, which is available to authorized users.

Avraham A. Levy

avi.levy@weizmann.ac.il

Yifat Tishler

yifat.tishler@weizmann.ac.il

Aviva Samach

aviva.samach@weizmann.ac.il

Ilana Rogachev

ilana.rogachev@weizmann.ac.il

Rivka Elbaum

rivka.elbaum@mail.huji.ac.il

1 Department of Plant and Environmental Sciences, Weizmann Institute of Science, P.O.B. 26, Rehovot 76100, Israel

2 The RH Smith Institute of Plant Sciences and Genetics in Agriculture, Hebrew University of Jerusalem, Rehovot 76100, Israel the cellulose fraction. There was a significant degree of variation among wheat lines in the enzymatic saccharification of the straw, following acid pretreatment. Interestingly, the straw from wild wheat had the highest degree of saccharification compared to domestic lines. These findings are of interest for the biofuel industry because they mean that wheat lines can be developed in which a significant amount of free soluble sugars can be easily extracted from straw without the need for costly pretreatment and enzymatic deconstruction. Moreover, the high FSS trait might be combined with the high enzymatic saccharification trait suggesting that wheat lines can be developed with a straw composition better adapted for biofuel production.

Keywords Lignocellulosic biomass · Bioethanol - Wheat straw $\cdot$ Free soluble sugars (FSS) $\cdot$ Water-soluble fraction (WSF)

\section{Introduction}

Wheat straw is a by-product left after the grain harvest, and thus has a great economic potential to generate bioethanol fuels worldwide. The annual global production of wheat grain in 2012 was estimated to be 670 million tons [1], and it keeps increasing each year. Assuming that the ratio between residues (straw) to grains is 1.3 [2], the residue weighs 887 million tons. Considering other applications of the wheat straw such as plowing back to the soil to prevent soil erosion or for tillage practices, burning, bedding, and forage for ruminants and more, it is estimated that half of this amount, i.e., 400 million tons, will remain unused and could be an available biomass for ethanol production [2]. According to biofuels-OECD-FAO Agricultural Outlook 2013-2022 [3], ethanol production is 
expected to increase by $67 \%$ over the next 10 years, and the forecast is to reach up to 168 billion liters by 2022 , most of it from sugarcane and grains. The prospect to produce bioethanol from these starch/sugar-rich crops exacerbates the food versus fuel conflict [3]. The feedstock for second generation biofuels produced from the lignocellulosic biomass of crops has the advantage of being cheap and abundant, and its production is not competing on agricultural lands or food unlike many other first generation biofuel feedstocks. Bioethanol produced from lignocellulosic biomass could satisfy a proportion of the increasing demand for liquid fuels [4], it can be used as sole fuel in cars with dedicated engines or as an additive (up to $30 \%$ ) with no engine modification requirements [2]. Still, technological progress is essential in order to increase the implementation of second generation biofuels and to reduce the processing cost to make it cost-competitive [5, 6]. The lignocellulosic biomass inside the plant cell wall is composed of three main components: lignin, hemicellulose, and cellulose. The conversion of lignocellulose biomass into ethanol requires three main steps: (i) pretreatment, which disrupts the connection between the carbohydrates and the lignin, depolymerizes and solubilizes the hemicellulose polymers, and exposes the cellulose and the hemicellulose for enzymatic degradation; (ii) saccharification, the enzymatic hydrolysis of the cell wall carbohydrates to their sugar monomers; (iii) microbial fermentation of the six carbon sugar monomers into ethanol [7]. During the development of cool season cereals (C3 plants) (e.g., wheat, barley, oats), water-soluble carbohydrates can accumulate in the stem and leaf sheath. They are mainly composed of fructans, sucrose, glucose, and fructose $[8,9]$. The phenomenon of nonstructural carbohydrate accumulation has been well documented in rice, in which the stem behaves as a tentative source of sugar translocation to the grains. Rice stem carbohydrates consist of starch, sucrose, and soluble reducing sugars (without fructans) [10]. Carbohydrates temporarily accumulate in wheat stems during the early reproductive growth phase, predominantly as water-soluble carbohydrates, and are subsequently remobilized during grain filling. This carbon accumulation becomes more important for grain yield in cereal crops under abiotic stress. In wheat plants undergoing drought stress, the carbon supply from photosynthesis is reduced due to both stomatal closure in the leaves and downregulation of genes involved in the Calvin Cycle [8]. Under these conditions, the stem water-soluble carbohydrates can contribute up to $50 \%$ of grain yield [8]. Wheat straw was used as a feedstock for bioethanol production [11-14], but successful engineering or breeding of straw that is well adapted to bioethanol production are still needed. Traits to improve include both the efficiency of enzymatic saccharification and the contents of sugars in the water-soluble fraction. Genetic engineering of wheat remains problematic due to the technical difficulty at performing transformation and because regulations for genetically modified wheat will probably be more draconian than for other crops not directly consumed by humans. On the other hand, there is a great biodiversity that has not been harnessed for improving straw as a feedstock for biofuel, and that could be used by breeders without regulatory hurdles. The wheat group (Aegilops and Triticum species) includes several species whose chromosomes can recombine with their homologs or homeologs in bread or durum wheat [15]. This enables the transfer of genes from these species into modern cultivars.

In this work, we have screened wheat biodiversity to identify lines whose straw is well adapted as a feedstock for bioethanol production. We screened a core collection of 48 wheat lines representing a broad spectrum of genetic variation, including wild and domestic lines, tetraploid and hexaploid, as well as traditional lines or modern varieties. The straw composition was analyzed, including the main components of the water-soluble fraction, such as starch, fructans, or free soluble sugars and also the cell wall components, such as cellulose, hemicellulose, and lignin [9]. We identified wheat lines with a high concentration of free soluble sugars (FSS) in the straw. Fructose and glucose were the two major sugars in this fraction. Such lines produce significant yield of fermentable sugars without the need for harsh pretreatment or for enzymatic deconstruction. In addition, we identified variation among wheat lines in the efficiency of enzymatic saccharification of the straw, following acid pretreatment.

\section{Materials and Methods}

\section{Plant Material and Growth Conditions}

The 48 wheat lines were grown in three replicates, each in a different block. Plants were grown in 5-1 pots, each pot corresponding to a different replica. All the 48 lines and their replicates were grown under the same conditions, in winter 2012, in a net house at the Weizmann Institute, Rehovot, Israel. The 48 lines of wheat were from six different genetic groups (Online Resource 1): Tetraploid wild emmer wheat (Triticum turgidum var. diccocoides, TTD), tetraploid domesticated emmer wheat (Triticum turgidum var. diccocum, TTC), tetraploid durum wheat (Triticum turgidum var. durum, TTR) both landrace (traditional) and modern and hexaploid bread wheat (Triticum aestivum var. aestivum, TAA) both landrace and modern [15]. Harvested stems and leaves from three upper nodes of the wheat straw were ground and dried at $60^{\circ} \mathrm{C}$.

\section{WSF Extraction}

A volume of $1.5 \mathrm{ml} \mathrm{DW}$ was added to $25 \mathrm{mg}$ of dried and ground wheat straw in tube 1 . The sample was boiled for $1 \mathrm{~h}$ and centrifuged at $10,000 \mathrm{rpm}$ for $5 \mathrm{~min}$. A volume of $1.3 \mathrm{ml}$ 
of the supernatant was transferred into tube 2, was freezedried, and weighed.

\section{DNS (3,5-Dinitrosalicylic Acid) Assay for Reducing Sugar Measurements}

The concentration of the reducing sugars $[\mathrm{mM}]$ was determined using the 3,5-dinitrosalicylic acid (DNS) assay using glucose as standard for the calibration curve. Each sample was diluted 20× with DDW and vortexed. Calibration was made by preparing glucose standards. A $20-\mathrm{mM}$ glucose solution was diluted in DDW to obtain final concentrations of glucose from 0 to $5 \mathrm{mM}$. Then, 150- $\mu$ l DNS [16] reagent was added to $100 \mu \mathrm{l}$ of the diluted hydrolyzate and to the glucose standards. The standards and samples were boiled for $5 \mathrm{~min}$. The absorption (OD) was read at $540 \mathrm{~nm}$. A calibration curve was plotted from the glucose standards and used to find the concentration of reducing sugars $[\mathrm{mM}]$ in the experimental samples.

\section{Determination of FSS and Starch Quantities in the WSF}

To quantify the free reducing sugars in the WSF, the DNS assay was carried out for half of the WSF, without any pretreatment. The second half was treated with $\alpha$ amylase and pullulanase enzymes (see cell wall isolation). The amount of reducing sugars in the untreated WSF was subtracted from this treatment to obtain the net amount of glucose obtained by starch enzymatic hydrolysis.

\section{Determination of Fructan Quantities in the WSF}

The amount of fructans was estimated by measuring the total amount of reducing sugars using the DNS assay before and after hydrolysis with $1 \% \mathrm{H}_{2} \mathrm{SO}_{4}$ for $1 \mathrm{~h}$ at $121{ }^{\circ} \mathrm{C}$. Reducing sugars generated due to the $\mathrm{H}_{2} \mathrm{SO}_{4}$ treatment would originate from fructan hydrolysis.

\section{Thermo-Gravimetric Analysis}

Thermogravimetric analysis (TGA) [17] of the samples was performed using the SDT Q600 V8.3 Build 101 thermal analyzer. Samples of about 7-10 mg were taken from the dried and ground straw for analysis at a temperature ranging between $23^{\circ}$ and $800{ }^{\circ} \mathrm{C}$ with a heating rate of $10{ }^{\circ} \mathrm{C} / \mathrm{min}$ and a flow of nitrogen at $100 \mathrm{ml} / \mathrm{min}$. The crucible used was of alumina (A12O3), which also served as a reference for the sample. From this, we obtained the data to determine the percent of weight loss for each sample and first derivative of the thermogram (DTG) which indicates the temperature of decomposition for the components in the wheat straw.

\section{Ion Chromatography Analysis for WSF}

The WSF was analyzed by high-performance anion-exchange chromatography [18] coupled to pulsed amperometric detection (HPAEC-PAD) using an Dionex ICS3000 system equipped with a CarboPac PA1 column coupled to a CarboPac PA1 guard column (both from Dionex). The amperometric detector was equipped with a disposable gold electrode (Dionex), and the standard carbohydrate waveform recommended by the manufacturer was applied. Injection volume was set at $10 \mu \mathrm{l}$ for all samples. Flow was set at $1 \mathrm{ml} / \mathrm{min}$. Sugars were eluted using two methods: gradient elution for the assignment of the differential compounds and isocratic elution for the absolute quantification of glucose, fructose, and sorbitol in WSF. Eluent A was DDW, eluent B was $0.1 \mathrm{M} \mathrm{NaOH}$. Gradient elution method was adopted from Couturier et al. [18]. Gradient program started from isocratic elution at $20 \%$ $\mathrm{B}$ for $20 \mathrm{~min}$; then, a linear gradient to $100 \% \mathrm{~B}$ was applied for $0.2 \mathrm{~min}$, isocratic elution at $100 \% \mathrm{~B}$ for $14.8 \mathrm{~min}$ to flush the column, linear gradient to $20 \% \mathrm{~B}$ for $0.2 \mathrm{~min}$ and reconditioning of the column at $20 \% \mathrm{~B}$ for $14.8 \mathrm{~min}$ (total run time was $50 \mathrm{~min}$ ). Peak assignment was based on the analysis of individual standards: glucose, fructose, sorbitol, mannose, arabinose, galactose, ribose (Sigma). Isocratic elution method: $50 \%$ A-50 \% B for 20 min. External calibration curves were prepared for fructose in the range of $1-100 \mu \mathrm{g} / \mathrm{ml}$, for glucose $1-25 \mu \mathrm{g} / \mathrm{ml}$, for sorbitol $1-15 \mu \mathrm{g} / \mathrm{ml}$.

\section{Enzymatic Hydrolysis of Acid-Pretreated Samples by Commercial Cellulase Preparation}

An acid pretreatment protocol [19] was carried out using 1-ml $1 \% \mathrm{H}_{2} \mathrm{SO}_{4}$ on 50 -mg ground straw in 2-ml tubes. Each tube was autoclaved at $121{ }^{\circ} \mathrm{C}$ for $20 \mathrm{~min}$ (overall cycle length $90 \mathrm{~min}$ ). After 4 washes with $1 \mathrm{ml} \mathrm{DDW}$ and one wash with 1 -ml acetate buffer ( $50 \mathrm{mM}, \mathrm{pH}=4.9)$, enzymatic hydrolysis of cellulose was performed. We added $1 \mathrm{ml}$ of acetate buffer $(50 \mathrm{mM}, \mathrm{pH}=4.9)$ in the presence of $32 \mu \mathrm{l}$ Celluclast and $8 \mu \mathrm{l}$ $\beta$-glucosidase (final enzyme loading is $25 \mathrm{mg}$ cellulase proteins per $1 \mathrm{~g}$ of dry sample). For this purpose, a commercial cellulase preparation (Sigma) supplemented with $\beta$ glucosidase (Sigma) was used. The tubes were placed in a shaker incubator at $50{ }^{\circ} \mathrm{C}$ and were agitated at $150 \mathrm{rpm}$ for $24 \mathrm{~h}$. The concentration of the reducing sugars $[\mathrm{mM}]$ generated by the enzymatic hydrolysis after $24 \mathrm{~h}$ was determined using the DNS assay.

\section{Cell Wall Component Analysis}

\section{Cell Wall Isolation}

Dried wheat straw was ground, weighed, and washed in $70 \%$ ethanol, vortexed and centrifuged at 10,000 rpm for $10 \mathrm{~min}$, 
and then the supernatant was aspirated. The pellet was resuspended in chloroform/methanol (1:1 v/v solution), centrifuged at 10,000 rpm, decanted, and resuspended in acetone. The solvent was evaporated, and the pellet was resuspended in sodium acetate buffer ( $\mathrm{pH}$ 5.0), $\alpha$-amylase (Sigma), and pullulanase (Sigma) in order to remove starch from the pellet. The pellet was washed three times with DDW. Acetone was added, and the pellet was air-dried [20].

\section{Lignin Content}

Lignin content was measured using the acetyl bromide soluble lignin (ABSL) method [20, 21]. The dried cell wall was weighed into a volumetric flask, and acetyl bromide solution (25\% v/v acetyl bromide in glacial acetic acid) was added. The acetyl bromide solution together with the cell wall was heated for $3 \mathrm{~h}$ at $50{ }^{\circ} \mathrm{C}$. Sodium hydroxide (Sigma) and hydroxylamine hydrochloride (Sigma) were added and diluted with glacial acetic acid. In order to measure the ABSL, $200 \mu 1$ of the solution was pipetted into a UV specific 96-well plate that was read in an ELISA reader at $280 \mathrm{~nm}$. The percentage of ABSL was determined using an appropriate coefficient (wheat straw $=17.5$ ) [22] with the following formula:

$\% \mathrm{ABSL}=\frac{\text { absorbance }}{(\text { Coeff } \times 0.539 \mathrm{~cm})} \times \frac{(10 \mathrm{ml} \times 100 \%)}{\text { weight }(\mathrm{mg})}$

$0.539 \mathrm{~cm}$ is the path length of the plate; weight is the cell wall weight in $\mathrm{mg}$.

\section{Crystalline Cellulose Content}

The dried cell wall was weighed into a tube. Updegraff [23] reagent (acetic acid/nitric acid/water, 8:1:2 v/v) was added, vortexed, and heated at $100{ }^{\circ} \mathrm{C}$ for $30 \mathrm{~min}$, when only crystalline cellulose remains insoluble in the pellet. Samples were cooled, centrifuged, washed with DDW and acetone, and airdried. The supernatant was discarded. The crystalline cellulose content was quantified after being completely hydrolyzed into glucose with the colorimetric Antherone assay [24]. For that purpose, $72 \%$ sulfuric acid was added and incubated at room temperature for $30 \mathrm{~min}$. The assay was performed in a 96-well plate. A glucose standard curve was prepared using 1$\mathrm{mg} / \mathrm{ml}$ glucose stock in order to create standard solutions of 0 , $2,4,6,8$, and $10 \mu \mathrm{g}$ glucose in $100 \mu \mathrm{LDW}$. From the samples, $2 \mu \mathrm{l}$ was added to $98 \mu \mathrm{l}$ DDW. To this, $200 \mu \mathrm{l}$ of freshly prepared Anthrone reagent was added. By heating the plate at $80^{\circ} \mathrm{C}$, glucose containing samples turn from yellow into blue green. Absorption (OD) was read at $625 \mathrm{~nm}$. Glucose (and hence crystalline cellulose content) was calculated based on the absorbance compared to the standard curve established on the same plate.

\section{Results and Discussion}

\section{WSF Composition in 48 Wheat Lines}

In order to determine the distribution of sugars in wheat straw, we fractionated it into a water soluble and a nonsoluble fraction (Fig. 1). The WSF was extracted by boiling in water which constitutes a simple and low-cost extraction of free sugars, starch, and fructans that could be used as a feedstock for second generation biofuel. Free sugars are readily available for fermentation, and starch could be hydrolyzed using established enzymatic protocols. In order to identify wheat lines with high WSF, we screened 48 lines of wheat from

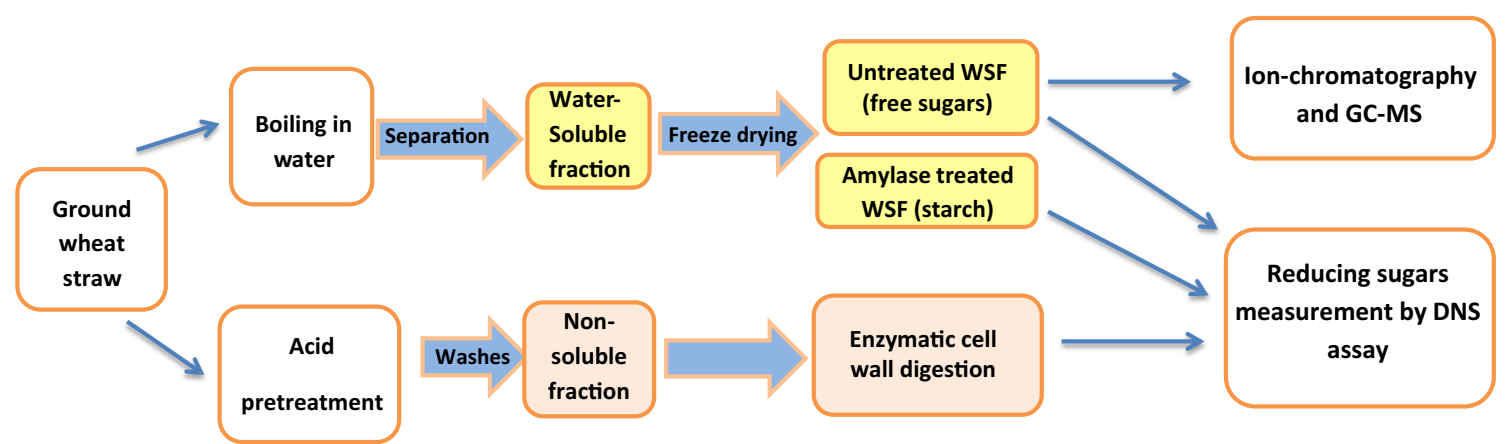

Fig. 1 Fractionation of wheat straw into potential biofuel producing components. This flow chart describes the procedure we used for analysis and comparison of fermentable components in straw of wheat lines. Wheat straw used for analysis was ground, dried, and weighed. To extract the FSS, water was added and the sample was boiled. After boiling, we separated the supernatant (water-soluble fraction-WSF) from the pellet and then freeze-dried. The WSF was then split into untreated or starch hydrolysis treatment. The untreated WSF was analyzed by DNS assay to determine its reducing sugar content (FSS), or by ion chromatography and GC-MS to determine which free sugars consisted in this fraction. Starch hydrolysis potential in the water-soluble fraction was determined by DNS measurement of the amylase-treated WSF. In parallel, the ground wheat straw was weighed and pretreated with acid, and then washed. The cellulose hydrolysis potential was determined by DNS measurement of the nonsoluble fraction after enzymatic cell wall digestion by cellulase and $\beta$-glucosidase 
various stages in wheat evolution, starting with wild emmer wheat, early domesticated types ( $T$. dicoccum), tetraploid and hexaploid landraces, and modern durum and bread wheat cultivars (Online Resource 1). All lines were grown under the same conditions and in three replicas (see "Materials and Methods"). In each line, we measured the amount of FSS released from straw after boiling (see "Materials and Methods") and the amount of reducing sugars released from starch after enzymatic digestion. For eight selected lines, we also measured the amount of reducing sugars released from fructans after acid treatment of the WSF. These three components together comprising the WSF.

WSF starch and FSS were measured in the 48 wheat lines (Fig. 2). Lines TAA1, TTR6, TTR52, and TTR42 had high FSS values with an average concentration of $28.3 \mathrm{mM}$ reducing sugars, and TAA48, TAA55, TTC8, and TTD168 had low sugar release values with an average concentration of $4 \mathrm{mM}$ reducing sugars (Fig. 2). The amount of starch ranged from 0 to $22 \mathrm{mM}$. We found significant differences between the lines (Online Resource 2a, b). Interestingly, the cumulative amount of reducing sugars, from the FSS and the starch, was significantly different between the evolutionary groups: it seems that the domesticated types, either from durum or bread wheat, had a significantly higher amount of sugars in the WSF compared to the wild emmer wheat group (TTD) which has the lowest WSF (Online Resource 3). It will be interesting to find out whether this reflects a more efficient translocation of sugars from the stem and leaves to the grain in the wild or a more effective production of sugars under domestication. Four lines with high and four lines with low FSS concentration were selected for further analysis. The FSS amounts of the two groups were significantly different (ANOVA $p$ value $0.0024)$. The presence of fructans in the WSF was measured in these 8 lines (see "Materials and Methods"). Results showed no difference in reducing sugars before and after the acid treatment. Therefore, we conclude that the WSF does not contain fructans (Online Resource 4).

\section{Free Sugar Monomers Comprising the FSS of Wheat Straw}

The FSS was analyzed by ion chromatography in order to determine sugar composition in the 8 lines analyzed. Results obtained from ion chromatography showed similar profiles of peaks; however, there were strong differences in the relative amount of specific peaks in the high- and low-sugar lines (Fig. 3a). In order to identify the substances of the differential peaks, we used seven sugar monomer standards. The differential peaks corresponded to fructose, glucose, and sorbitol (Fig. 3b). Quantification of sugars was done against the corresponding standards and is shown in Fig. 3c. Fructose is the most abundant free sugar monomer of the WSF in both

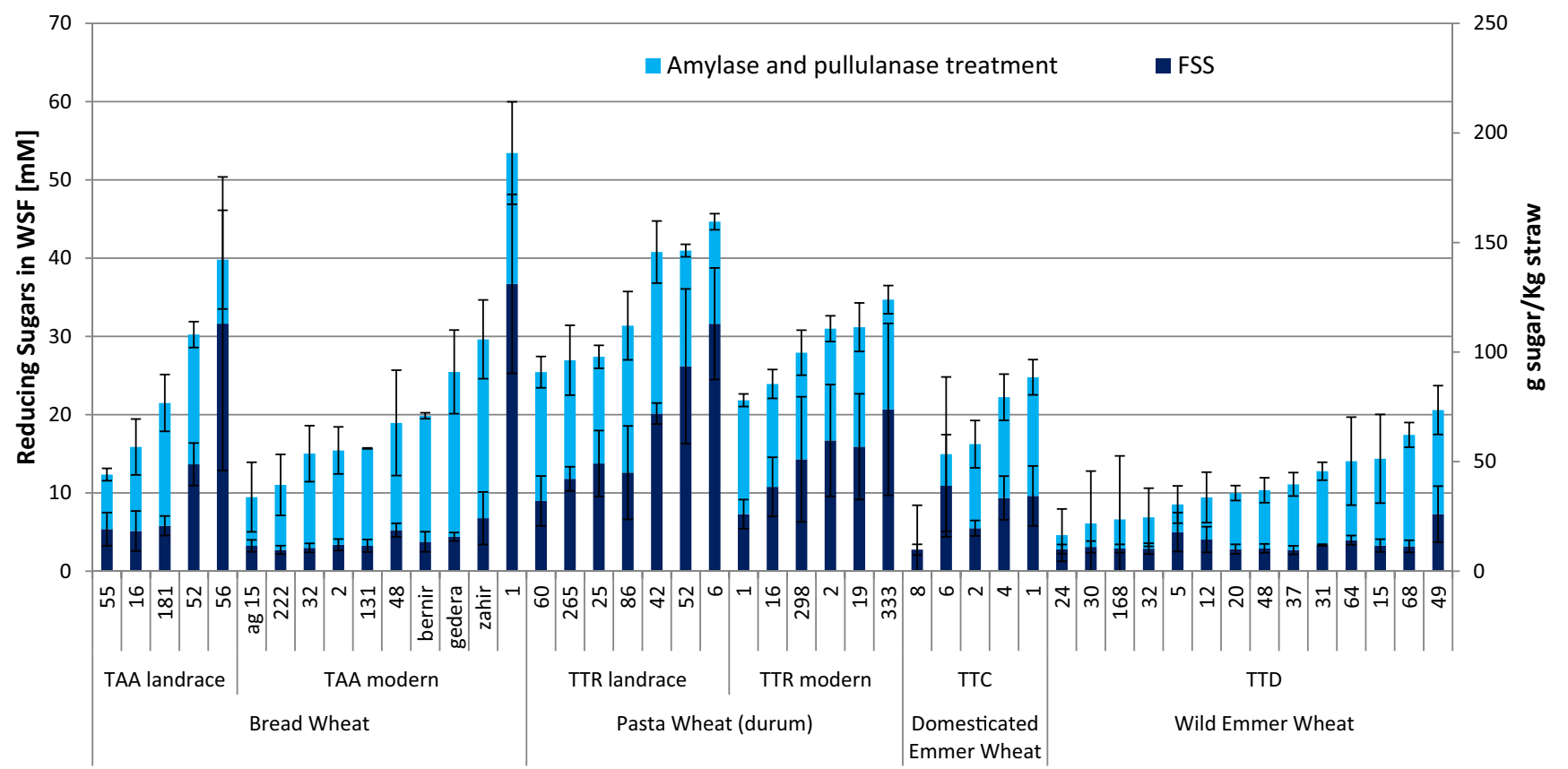

Fig. 2 Reducing sugars in the water-soluble fraction (WSF) of wheat straw. TAA - Triticum aestivum var: aestivum; TTR—Triticum turgidum var. durum; TTC-Triticum turgidum var. dicoccum; TTD-Triticum turgidum var. dicoccoides; landrace - domesticated noncommercial line grown by local farmers; modern-commercial line. The free soluble sugar (FSS) fraction was obtained without any enzymatic treatment (dark blue), and the starch fraction was determined by measuring reducing sugars in the WSF after amylase treatment (light blue), DNS assay was used to determine the free reducing sugar amounts in each fraction. Each column represents the average and standard error of three biological repeats 
a

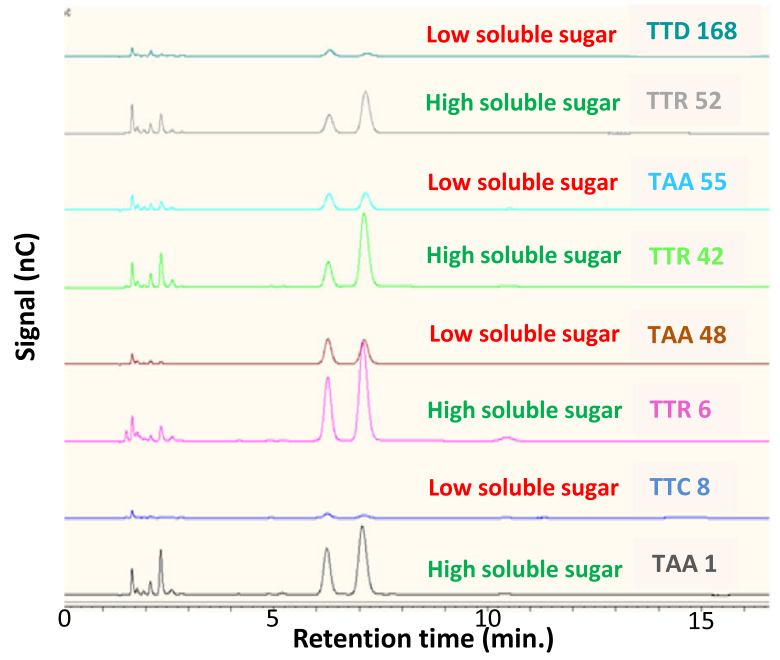

C

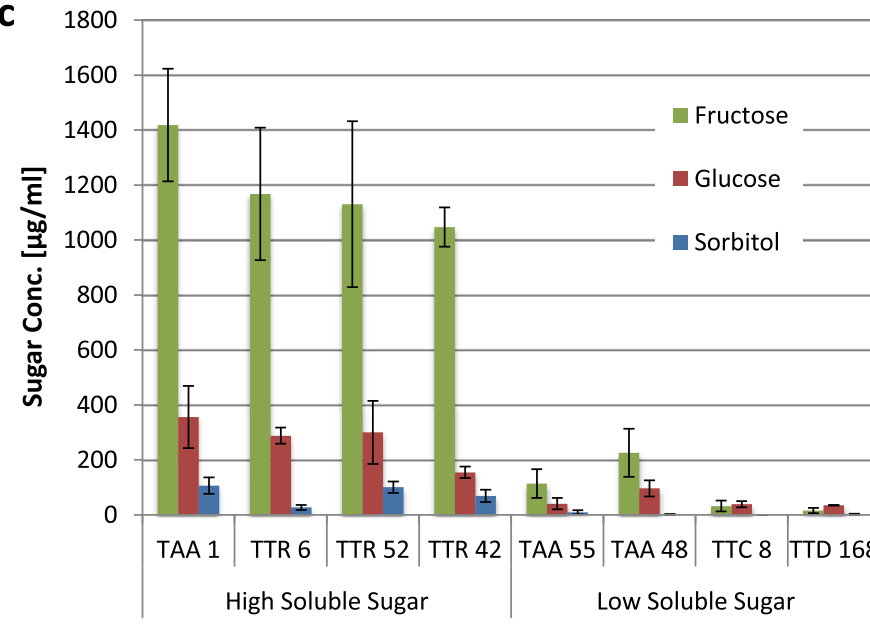

Fig. 3 Identification of free sugar types in the WSF by ion chromatography. a Differential peaks obtained by the ion chromatography of lines with high and low free soluble sugar concentration; b identification of sugar peaks in the samples according to sugar standards; c fructose, glucose, and

groups, and it is also the main component differentiating the FSS composition of high and low soluble sugar groups. Identification of the free sugars that comprise the WSF was also conducted using GC-MS and confirmed the ion chromatography results (Online Resource 5). These sugars were quantified by comparison of their retention time and mass spectra with sugar standards analyzed together with the samples and were relatively quantified by normalization to ribitol internal standard (Online Resource $5)$. There were small differences in the quantification of free sugars (g sugar $/ \mathrm{kg}$ straw) between the ion chromatography data (Fig. 3c) and the DNS method (Fig. 2); nevertheless, we can conclude that the amount of monomeric sugars ranges between 100 and $200 \mathrm{~g} / \mathrm{kg}$ straw in the high-sugar lines, compared with values below $30 \mathrm{~g} / \mathrm{kg}$ straw in the low-sugar lines. Moreover, fructose is the main component of the free sugars in the WSF.

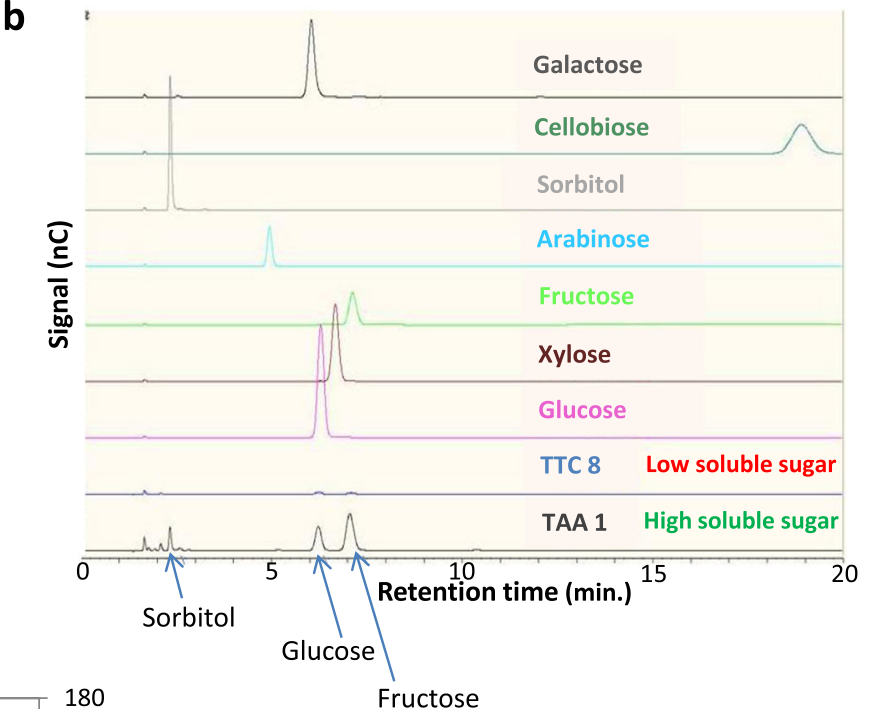

160

140

120

100

80

$60 \stackrel{\text { 矛 }}{\text { bo }}$

40

20

0

sorbitol concentrations and amounts in the WSF determined by ion chromatography. The eight extreme lines of the two sugar release groups were compared. Each column represents the average and standard error of three biological repeats

\section{Wheat Straw Main Components by Pyrolysis}

The main components in wheat straw were also analyzed using thermogravimetric analysis (TGA). Figure 4 shows the differential thermogravimetric (DTG) curves which give the rate of weight loss for each temperature, with water being lost from the sample around the $60^{\circ} \mathrm{C}$ peak. The cell wall in wheat straw undergoes a temperature-dependent decomposition process. The hemicellulose decomposition peak is at $260^{\circ} \mathrm{C}$, and the cellulose decomposition peak is at $330{ }^{\circ} \mathrm{C}$. Overall, the majority of the sugar biomass decomposes at $250-350{ }^{\circ} \mathrm{C}$. Lignin has a spectrum of decomposition between 250 and $800^{\circ} \mathrm{C}$ according to its several different structures that decompose at different temperatures, although most of the decomposition occurs between $360^{\circ} \mathrm{C}$ and $800^{\circ}$ [17].

A clear difference was obtained between high and low FSS lines (Fig. 4a). The high FSS lines (in green) show a peak at 


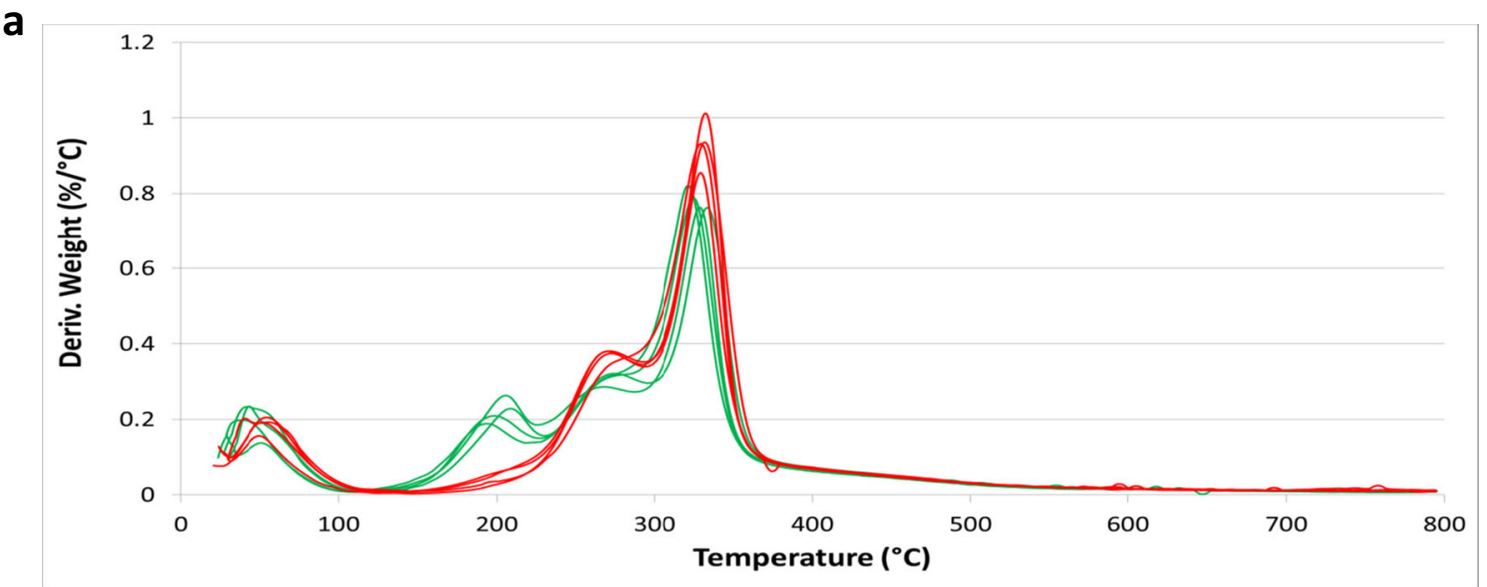

b

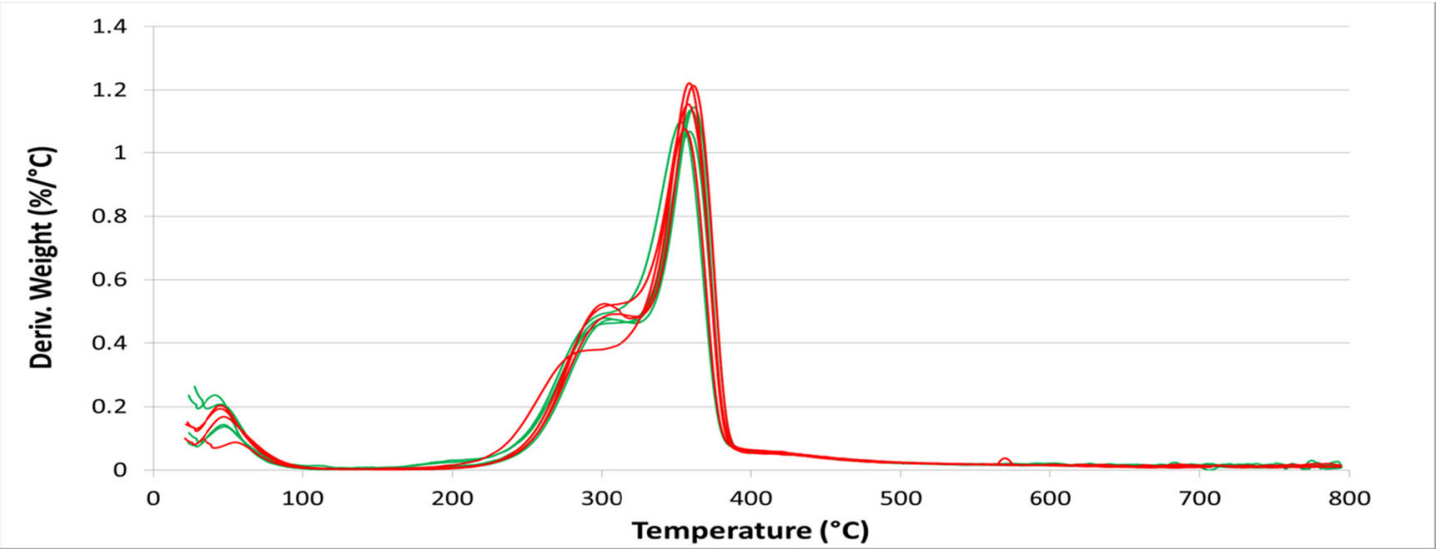

High soluble sugar lines

Low soluble sugar lines

Fig. 4 Thermal gravimetric analysis in selected wheat lines. Wheat straw TGA derivative (DTG) of eight extreme lines from high and low FSS groups. a Decomposition rates of straw biomass samples; b decomposition rates of the nonsoluble fraction samples remaining after
WSF extraction. Known decomposition peaks: water- $60{ }^{\circ} \mathrm{C}$; hemicellulose $-260{ }^{\circ} \mathrm{C}$; cellulose $-330{ }^{\circ} \mathrm{C}$; lignins $-250{ }^{\circ} \mathrm{C}$ to $800{ }^{\circ} \mathrm{C}$ The peak at $200{ }^{\circ} \mathrm{C}$ seen in a disappeared after the WSF extraction in $\mathbf{b}$
$200{ }^{\circ} \mathrm{C}$ that is almost absent in the low FSS lines (in red). The WSF was extracted from the samples, and the dried nonsoluble fraction was analyzed by TGA. As expected, the peak at $200{ }^{\circ} \mathrm{C}$ disappeared in all of the samples after the WSF extraction (Fig. 4b). The other peaks, corresponding to hemicellulose, cellulose, and lignin, did not show significant difference between the lines.

\section{Differences Among 48 Wheat Lines in Enzymatic Hydrolysis After Acid Pretreatment}

Cellulose is a major source of sugars available from the pretreated wheat straw. Here, we used a commercially relevant acid pretreatment (see "Materials and Methods") on all of the 48 lines followed by enzymatic hydrolysis of the cellulose (Fig. 5). The amount of reducing sugars within the tested lines ranged between $67 \mathrm{mM}$ up to $110 \mathrm{mM}$. We found significant differences between the lines (Online Resource 2c). On average, the wild wheat group (TTD) showed higher levels of enzymatic saccharification compared to the other groups (Online Resource 6). This might be due to selection for a stiffer straw during domestication. It might be that this high digestibility trait could be restored in modern wheat varieties that are dwarf or semi-dwarf and where lodging is not problematic.

\section{Analysis of the Straw Cell Wall Main Components}

In order to test if there is a correlation between the sugar release (following acid pretreatment and enzymatic digestion) and the cell wall components, we measured lignin and crystalline cellulose in six high-sugar release lines (TAA 131,TAA 1, TAA Zahir, TAA Gedera, TTR 2, TAA55) and in six lowsugar release lines (TAA Bernir, TTD31, TTD 68, TTD 5, 


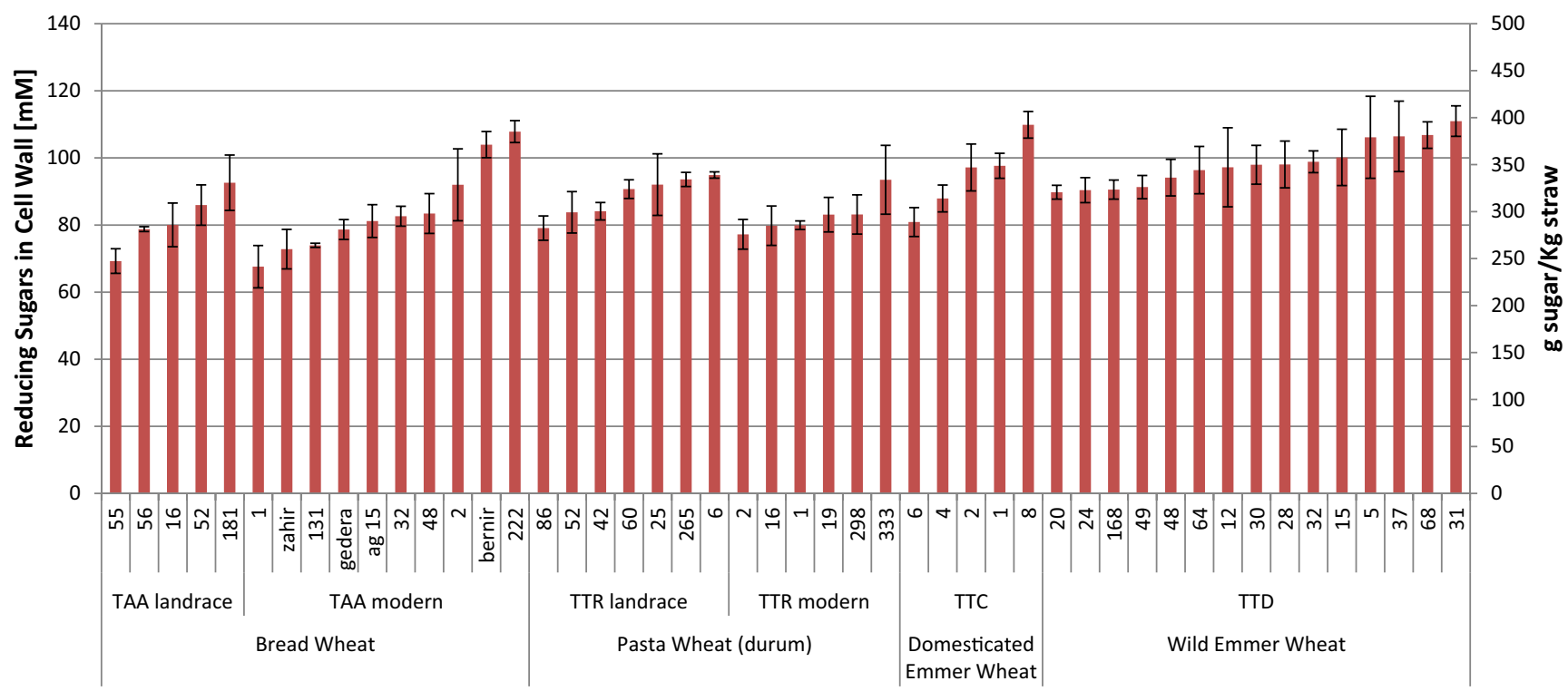

Fig. 5 Enzymatic sugar release from straw after acid pretreatment. TAA-Triticum aestivum var. aestivum; TTR-Triticum turgidum var. durum; TTC-Triticum turgidum var. dicoccum; TTD-Triticum

TTC 8, TAA 222). Lignin content in the cell wall of wheat straw ranged between 18 and $23 \%$, with no correlation to the high- and low-sugar release groups (Online Resource 7a). Crystalline cellulose content in the cell wall ranged between 27 and $37 \%$ (Online Resource 7b), with no correlation to the high- and low-sugar release lines. FSS amount in these lines also did not show any correlation to sugar release (Online Resource 7c).

\section{Conclusions}

We discovered much variation in the content of FSS in the straw among the wheat lines analyzed. Free sugars consist mostly of fructose and glucose and to a lesser extent of sorbitol, three monosaccharaides that are easy to ferment into ethanol using standard industrial yeast strains [25]. Extracting these sugars from the straw requires only grinding and boiling, with no need for costly pretreatment or enzymatic treatment for deconstruction. In quantitative terms, the potential availability of such resource for bioethanol production is very significant. Despite fluctuations with the methods (DNS, ion chromatography and GC-MS) and the lines, we consider for sake of simplicity that the FSS in the WSF of wheat straw could represent up to $10 \%$ of the biomass $\sim 100 \mathrm{~g} / \mathrm{kg}$. Considering the 400 million tons of straw (see "Introduction") that are available worldwide as a feedstock for biofuel, this means that 40 million tons of sugars could be extracted at low cost and converted to $\sim 20$ billion liters of ethanol. This value could be further increased, using glucose from the starch in the WSF, which is also easy to extract, requiring a simple amylase turgidum var. dicoccoides; landrace - domesticated noncommercial line grown by local farmers; modern-commercial line. Each column represents the average and standard error of three biological repeats

treatment, or alternatively amylase activity can be engineered in yeast [26]. Further studies should help determine if the high free sugar trait in the straw is desirable or is associated negatively to agronomic performances, and genetic and genomic analysis should be helpful to determine the molecular basis for this phenotype. In Sorghum, high sugar in the stems and high grain yield can coexist [27]; therefore, it might also be possible in wheat. The ease of extraction of the WSF opens the prospect for simple scenarios, such as processing of the wheat straw on the farms where wheat is harvested, providing farmers with a means of producing ethanol on-site. Finally, the enzymatic saccharification of the nonsoluble fraction, i.e., the cell wall, after pretreatment, showed limited but significant differences $(\sim 30 \%)$ between lines (Fig. 5). Increasing both the WSF sugars and the efficiency of enzymatic saccharification in wheat straw would contribute to its improvement as a feedstock for bioethanol production.

Acknowledgments We would like to thank Prof. Moshe Feldman (Weizmann Institute of Sciences) for providing seeds of the wheat collection and for advice and Prof. Asaph Aharoni (Weizmann Institute of Sciences) for advice with the sugar composition analysis. We thank Dr. Eli Morag and Dr. Michael Loelovich (Designer Energy) for help with the cellulose hydrolysis. We thank Dr. Miron Abramson and Tali Eylat (Futuragene) for help with the cell wall component analysis. We also thank Ilan Bessudo for help with preparation of straw samples for analysis and Dr. Zvi Tamari for help with sugar composition analysis. This work was supported by a grant from the ICORE foundation, solar fuel project and by the Leona and Harry Helmsley charitable trust to A.A.L. YT was supported by the Ministry of Science and Technology, Israel. 
Open Access This article is distributed under the terms of the Creative Commons Attribution 4.0 International License (http:// creativecommons.org/licenses/by/4.0/), which permits unrestricted use, distribution, and reproduction in any medium, provided you give appropriate credit to the original author(s) and the source, provide a link to the Creative Commons license, and indicate if changes were made.

\section{References}

1. FAOSTAT Gateway_world wheat production 2012. http://faostat3. fao.org/faostat-gateway/go/to/download/Q/QC/E. Accessed 28 Jun 2014

2. Talebnia F, Karakashev D, Angelidaki I (2010) Production of bioethanol from wheat straw: an overview on pretreatment, hydrolysis and fermentation. Bioresour Technol 101:4744-4753. doi:10. 1016/j.biortech.2009.11.080

3. OECD-FAO Agricultural Outlook- Biofuels. http://www.oecd.org/ site/oecd-faoagriculturaloutlook/biofuels.htm. Accessed 15 Jun 2014

4. Naik SN, Goud VV, Rout PK, Dalai AK (2010) Production of first and second generation biofuels: a comprehensive review. Renew Sustain Energy Rev 14:578-597. doi:10.1016/j.rser.2009.10.003

5. To H, Sen S, Charles M (2014) Economic issues in the liquid biofuels industry. In: Domingos Padula A, Silveira dos Santos M, Benedetti Santos OI, Borenstein D (eds) Liq. Biofuels Emergence, Dev. Prospect. SE -1. Springer, London, pp 1-23

6. Walker GM (2011) 125th anniversary review: fuel alcohol: current production and future challenges. J Inst Brew 117:3-22

7. Kristensen JB, Thygesen LG, Felby C et al (2008) Cell-wall structural changes in wheat straw pretreated for bioethanol production. Biotechnol Biofuel 1:5. doi:10.1186/1754-6834-1-5

8. Xue G-P, McIntyre CL, Jenkins CLD et al (2008) Molecular dissection of variation in carbohydrate metabolism related to watersoluble carbohydrate accumulation in stems of wheat. Plant Physiol 146:441-454. doi:10.1104/pp.107.113076

9. Chatterton NJ, Watts KA, Jensen KB et al (2006) Nonstructural carbohydrates in oat forage. J Nutr 136:2111S-2113S

10. Park JY, Kanda E, Fukushima A et al (2011) Contents of various sources of glucose and fructose in rice straw, a potential feedstock for ethanol production in Japan. Biomass Bioenerg 35:3733-3735. doi:10.1016/j.biombioe.2011.05.032

11. Pensupa N, Jin M, Kokolski M et al (2013) A solid state fungal fermentation-based strategy for the hydrolysis of wheat straw. Bioresour Technol 149:261-267. doi:10.1016/j.biortech.2013.09.061

12. Ballesteros I, Negro MJ, Oliva JM et al (2006) Ethanol production from steam-explosion pretreated wheat straw. Appl Biochem Biotechnol 129-132:496-508. doi:10.1385/ABAB:130:1:496

13. Erdei B, Barta Z, Sipos B et al (2010) Ethanol production from mixtures of wheat straw and wheat meal. Biotechnol Biofuel 3: 16. doi:10.1186/1754-6834-3-16
14. Chen H, Han Y, Xu J (2008) Simultaneous saccharification and fermentation of steam exploded wheat straw pretreated with alkaline peroxide. Process Biochem 43:1462-1466. doi:10.1016/j. procbio.2008.07.003

15. Feldman M, Bonjean A, Angus W (2001) The origin of cultivated wheat. World wheat book. Lavoisier Publ, Paris

16. Detns RC, Taken S, Co FS et al (1959) Use of Dinitrosalicylic acid reagent for determination of reducing sugar. Anal Chem 31:426428. doi:10.1021/ac60147a030

17. Jae J, Tompsett GA, Lin Y-C et al (2010) Depolymerization of lignocellulosic biomass to fuel precursors: maximizing carbon efficiency by combining hydrolysis with pyrolysis. Energy Environ Sci 3:358. doi:10.1039/b924621p

18. Couturier M, Haon M, Coutinho PM et al (2011) Podospora anserina hemicellulases potentiate the Trichoderma reesei secretome for saccharification of lignocellulosic biomass. Appl Environ Microbiol 77:237-246. doi:10.1128/AEM.01761-10

19. Tutt M, Kikas T, Olt J (2012) Influence of different pretreatment methods on bioethanol production from wheat straw. Agron Res 10:269-276

20. Foster CE, Martin TM, Pauly M (2010) Comprehensive compositional analysis of plant cell walls (Lignocellulosic biomass) part I: lignin. J Vis Exp. doi:10.3791/1745

21. Fukushima RS, Hatfield RD (2001) Extraction and isolation of lignin for utilization as a standard to determine lignin concentration using the acetyl bromide spectrophotometric method. J Agric Food Chem 49:3133-3139. doi:10.1021/jf010449r

22. Fukushima RS, Hatfield RD (2004) Comparison of the acetyl bromide spectrophotometric method with other analytical lignin methods for determining lignin concentration in forage samples. J Agric Food Chem 52:3713-3720

23. Updegraff DM (1969) Semimicro determination of cellulose in biological materials. Anal Biochem 32:420-424. doi:10.1016/S00032697(69)80009-6

24. Foster CE, Martin TM, Pauly M (2010) Comprehensive compositional analysis of plant cell walls (lignocellulosic biomass) part II: carbohydrates. J Vis Exp. doi:10.3791/1837

25. Wu X, Staggenborg S, Propheter JL et al (2010) Features of sweet sorghum juice and their performance in ethanol fermentation. Ind Crops Prod 31:164-170. doi:10.1016/j.indcrop.2009.10.006

26. Rothstein SJ, Lazarus CM, Smith WE et al (1984) Secretion of a wheat $\alpha$-amylase expressed in yeast. Nature 308:662-665. doi:10. 1038/308662a0

27. Calviño M, Messing J (2012) Sweet sorghum as a model system for bioenergy crops. Curr Opin Biotechnol 23:323-329. doi:10.1016/j. copbio.2011.12.002

28. Nguyen SK, Sophonputtanaphoca S, Kim E, Penner MH (2009) Hydrolytic methods for the quantification of fructose equivalents in herbaceous biomass. Appl Biochem Biotechnol 158:352-361. doi:10.1007/s12010-009-8596-x 